\title{
Lipoatrofia semicircular. Nuestra experiencia dos años después (2007-2009)
}

\section{Semicircular Lipoatrophy. Our experience two years later (2007-2009)}

\author{
Antonio Ortega Díaz de Cevallos \\ Servicio de Medicina Interna. \\ Hospital Central Fraternidad-Muprespa. Madrid \\ Fernando Cuevas Moreno \\ Servicio de Medicina Interna. \\ Hospital Central Fraternidad-Muprespa. Madrid \\ Eduardo Aguado Posadas \\ Servicio de Medicina Interna. \\ Hospital Central Fraternidad-Muprespa. Madrid \\ Correspondencia: \\ Dr. Antonio Ortega Díaz de Cevallos. \\ Hospital Central Fraternidad Muprespa. \\ Servicio de Medicina Interna. \\ Paseo de la Habana 83-85. 28036. Madrid. España. \\ Teléf: + 34913608 800/ +34913608478. \\ E-mail: aortega@fraternidad.com
}

Resumen

Fundamento y objetivos: Describir y analizar la presentación clínica y la evolución tras la corrección de determinados factores ambientales, de nuestra serie de casos de lipoatrofia semicircular en el entorno laboral, con especial atención a la resolución de la lesión y valorar la posible asociación con otras patologías desde el punto de vista etiológico o que pudieran formar parte de un síndrome más complejo.

Pacientes y método: Trabajadores atendidos entre la primavera de 2007 y el verano de 2009. En la primera consulta se efectúa historia clínica detallada, con medición y localización de la lesión, realización de analítica y de ecografía de la zona supuestamente afecta. Posteriormente tras haberse tomado las medidas recomendadas en las empresas para eliminar la electricidad estática, se incluyen controles cada tres meses con anamnesis, exploración física y ecografía comparativas.

Resultados: El 96,7\% son mujeres, sin predominio de ninguna edad Todos trabajan en puestos con material informático y abundante cableado eléctrico. Las localizaciones, salvo 3 casos (2 en brazo y uno en región glútea), fueron a nivel de cara anterolateral de muslos y con valores medios de 69x20x73 cm. (altoxanchoxlargo). Evolutivamente a los 6 meses un 93\% de casos mostraban remisión clínica (62\% completa, $31 \%$ parcial); al año solamente 2 casos no han mostrado ninguna variación habiendo un $69 \%$ de resoluciones completas. Ningún caso ha presentado otra patología relacionada. En el primer año de control hubo 36 nuevos casos, en el segundo 22 y 4 en los 6 meses trascurridos del tercero.

Conclusiones: Las características clínicas son superponibles a las de otras series publicadas en cuanto a manifestaciones, tipo de trabajador afecto y predominio de presentación en mujeres. En cuanto a la evolución una vez modificado el entorno laboral, es evidente la tendencia a la resolución, mucho más rápida que en los casos antiguamente publicados en los que no se modificaba el entorno, en una gran proporción de los casos. Además estas correcciones han demostrado una gran eficacia frenando la aparición de nuevos 
casos. No se han apreciado otras manifestaciones patológicas que las estrictamente propias de lipoatrofia segmentaria.

(Med Segur Trab (Internet) 2009; 55 (217): 33-40)

Palabras clave: Lipoatrofia semicircular, manifestaciones clinicas, evolución, entorno laboral, factores ambientales.

Semicircular Lipoatrophy. Our experience two years later (2007-2009).

\section{Abstract}

Background and objectives: To describe and to analyze the clinical presentation and the evolution after variation of certain environmental factors, of our series of cases of semicircular lipoatrophy in job environment, with special attention to the resolution of the injury and to value the possible association with other patologies from the ethiologic point of view or if it could be part of a more complex syndrome.

Patients and method: Workers attended between spring of 2007 and summer of 2009. In the first visit, we made a standardized history performing physical examination with measurement and location of the injury, laboratory parameters tests and ultrasonographic study of the presumptively affected zone. Subsequently, after having been taken the measurements recommended in companies to eliminate the static electricity, quarterly controls are included with anamnesis, physical exploration and ultrasonographic comparatives.

Results: $96,7 \%$ are women, without predominance of any age. They all work at desks with informatic material and abundant electric wired up. The locations, except for 3 cases ( 2 in arm and one in bottom region), were at the level of the anterolateral face of thighs and with average values of $69 \times 20 \times 73 \mathrm{~cm}$. (heightxwidthxlength). Evolvely 6 months later $93 \%$ of cases was showing clinical remiss (62\% complete, $31 \%$ partial); after one year only 2 cases have not showed any variation having a $69 \%$ of complete resolutions. None of the cases has presented any other related pathology. In the first year of control there were 36 new cases, in the second one 22 and in what takes of the third one 4.

Conclusions: The clinical characteristics are superimposable on those of other published series as for manifestations, type of affected worker and predominance of presentation in women. As for the evolution once modified the job environment, the trend is evidently to the resolution, much faster than in the published former cases where the environment had not been modified, in a great proportion of the cases. Furthermore these alterations have demonstrated a great efficiency stopping the appearance of new cases. No other pathological manifestations have been assessed than the strictly proper ones of the semicircular lipoatrophy.

(Med Segur Trab (Internet) 2009; 55 (217): 33-40)

Keywords: semicircularlipoatrophy, clinical manifestations, evolution, job environment, environmental factors. 


\section{INTRODUCCIÓN}

Se define la lipoatrofia semicircular (LS) como una depresión lineal de la superficie cutánea que se localiza habitualmente en la cara antero-externa del muslo, producida por pérdida del tejido adiposo, sin signos inflamatorios y con integridad completa de la piel y del músculo adyacentes. Se han descrito casos aislados de otras localizaciones como brazos, abdomen y tobillos.

Hasta hace pocos años se consideraba una entidad muy poco frecuente y así sigue siendo fuera del ámbito de la medicina laboral. En 1974 los Drs. Schwandtner y Münzberger relacionan por primera vez la enfermedad con cierto tipo de edificios ${ }^{1,2}$, observación confirmada posteriormente (1981) por Karkavitsas y Milla ${ }^{3}$. Es a partir de 1990 cuando se publican múltiples casos en los Países Bajos, Francia e Italia ${ }^{4-6}$ de forma progresiva hasta que, desde principios de 2007 se reconocen más de 650 casos en diversos edificios de distintas empresas españolas.

Se trata de una enfermedad de etiología desconocida hasta ahora en la que se han invocado factores genéticos (alteraciones en los cromosomas 1q, 9q y 10), raciales (lipodistrofia abdominal japonesa) o autoinmunes (Lupus, esclerodermia) en común con otras lipodistrofias primarias ${ }^{7-9}, \mathrm{y}$ factores relacionados con lipodistrofias adquiridas como la administración de ciertos medicamentos (antirretrovirales, vasopresina, insulina), traumatismos directos y microtraumatismos de repetición. ${ }^{10-14}$.

Desde 2003 en que Maes publica su hipótesis electromagnética ${ }^{15}$, se relaciona con efectos electromagnéticos/electroestáticos sobre el tejido graso, si bien no ha habido hasta el momento ninguna comprobación experimental al respecto. Según Maes, la acumulación de cargas eléctricas en los objetos, principalmente metálicos, producirían descargas repetidas al roce con los muslos (zona corporal más próxima al tablero de la mesa) lo cual estimularía la producción de citoquinas por parte de los macrófagos, dañando al adipocito. Estas descargas pueden estar por debajo del umbral de sensibilidad y en recientes mediciones efectuadas, las fuerzas del campo magnético no superan ningún límite recomendado pero siempre aparecen apreciablemente más altas en los puestos de trabajos de los trabajadores con LS que en los de los no afectados ${ }^{16}$.

La LS microscópicamente se caracteriza por una involución del tejido celular subcutáneo a expensas de un aumento en la reabsorción del tejido graso, con disminución en el número y el volumen de los adipocitos, que en estadíos finales puede quedar sustituido por tejido conectivo ${ }^{17-18}$.

Según el estudio de 900 casos realizado por el Dr. Bart L. Curvers ${ }^{19}$, la LS puede aparecer a cualquier edad, predominando en un $85 \%$ en el sexo femenino y afectaría aproximadamente a un $30 \%$ de los trabajadores de ciertos edificios modernos. Actualmente los edificios donde se están diagnosticando los nuevos casos de lipoatrofia, están caracterizados por presentar una carga electromagnética elevada con una baja humedad relativa, teniendo probablemente un ligero mayor riesgo los trabajadores que utilizaran superficies de asiento elevadas y con escaso uso del apoyo lumbar ${ }^{20-22}$ con un tiempo de exposición calculado entre 4 y 6 meses.

Se presenta la LS en pacientes en los que se observa una depresión lineal de entre 1 y $5 \mathrm{~mm}$ de profundidad, con entre 5 y $15 \mathrm{~cm}$ de longitud y unos $2 \mathrm{~cm}$ de anchura, situada a unos $72 \mathrm{~cm}$ del suelo y sin signo alguno de flogosis. Suele ser un hallazgo ocasional, sin otra sintomatología acompañante relacionada, y que había pasado inadvertido por el paciente que, por lo tanto, no puede precisar la duración de la patología con exactitud. El resto de la anamnesis y de la exploración física suele ser normal o con patología absolutamente independiente de la LS.

El diagnóstico es eminentemente clínico con la exploración por inspección y palpación de la lesión, que se puede completar con estudios por imagen como ecografía y $\mathrm{RM}^{23}$. Los estudios de laboratorio no muestran ningún hallazgo específico y la biopsia cutánea no suele ser precisa, ya que en los casos más dudosos se recomienda solamente 
la realización de controles evolutivos que harán evidente, o descartarán, la enfermedad en unas semanas.

El diagnóstico diferencial se plantea principalmente con la lipoatrofia anular que es unilateral, afecta igualmente a los miembros superiores que a los inferiores, se acompaña de artralgias, miopatías, neuralgias, edemas distales y no tiende a la resolución; y con las atrofias secundarias y autoinmunes (lupus, esclerodermia, medicamentosa, hipodermitis postraumática, paniculitis de Weber Christian).

\section{PACIENTES Y MÉTODO}

Hemos realizado un estudio clínico-evolutivo retrospectivo, basado en el análisis de los datos extraídos de una base formada con pacientes con presunto diagnóstico de LS.

Nuestro protocolo está constituido por una historia clínica detallada, con atención especial a las características de la lesión (altura de localización, amplitud, afectación perilesional etc.). A todos los pacientes se les ha efectuado también, ecografía de la zona afectada y analítica con hematología, bioquímica, enzimas, reactantes de fase aguda y marcadores de enfermedades autoinmunes, además de las determinaciones exigidas por otras patologías intercurrentes identificadas. Para el control evolutivo de la enfermedad se han revisado (al menos) a los 3, 6, 9 y 12 meses, clínica y ecográficamente, a todos los pacientes para identificar la aparición de nuevos signos o síntomas, posibles complicaciones o la resolución, total o parcial, tras corregir los factores implicados en la aparición de la enfermedad, principalmente en lo referido a la sobrecarga de electricidad estática y al grado de humedad relativa del aire ambiente.

\section{RESULTADOS}

De los 93 casos estudiados, 62 (66,67\%), fueron finalmente diagnosticados de lipoatrofia semicircular, en 58 casos hemos podido realizar seguimiento de al menos un año u obtener datos de remisión completa.

Todos trabajan en edificios altamente tecnificados y con mayoría de horas de su jornada laboral realizadas en mesas y escritorios con dispositivos electrónicos (ordenadores, impresoras, escáneres...) y abundante cableado eléctrico.

Una abrumadora mayoría de casos se da en mujeres (96,7\%) sin diferencias significativas por rangos de edad. En cuanto a la localización, todos los casos menos tres se situaban en los muslos de forma uni o bilateral (Figura 1) sin diferencias significativas; las excepciones tenían la LS, uno en un brazo además de en el muslo homolateral, el segundo en ambos brazos y el otro en región glútea (atribuido a que la paciente con frecuencia se semisentaba en el borde de la mesa).

Figura 1 - Distribución de la afectación en muslos.

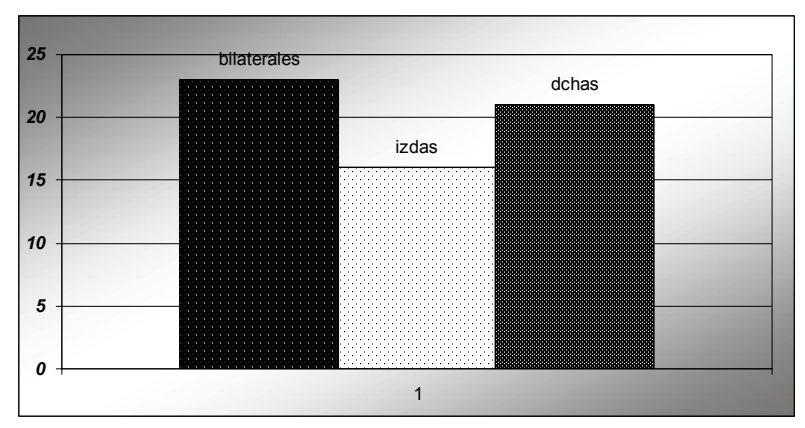

Lipoatrofia semicircular. Nuestra experiencia dos años después (2007-2009) 
Las características de la lesión vienen definidas por encontrarse a una altura del suelo que oscila entre 51 y $84 \mathrm{~cm}$ con una media de $69 \mathrm{~cm}$ (Figura 2). La altura de $72 \mathrm{~cm}$ se considera estándar en mesas y otro mobiliario de escritorio de oficinas.

Figura 2 - Distancia perpendicular lesión-suelo

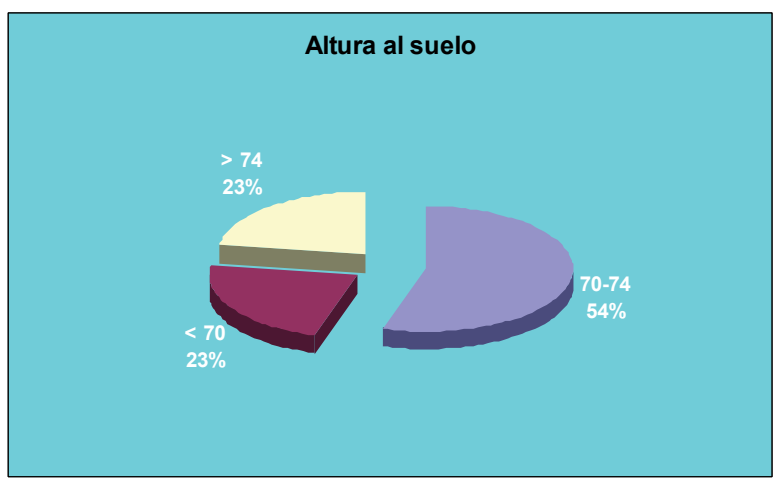

La anchura mostraba un rango de entre 10 y $45 \mathrm{~mm}$ (media de 19,67) situándose entre 10 y $20 \mathrm{~mm}$ en el $90 \%$ de los casos. La longitud ha sido de entre 20 y $180 \mathrm{~mm}$ (media de 73) (Figura 3).

Figura 3 - Longitud de la lesión

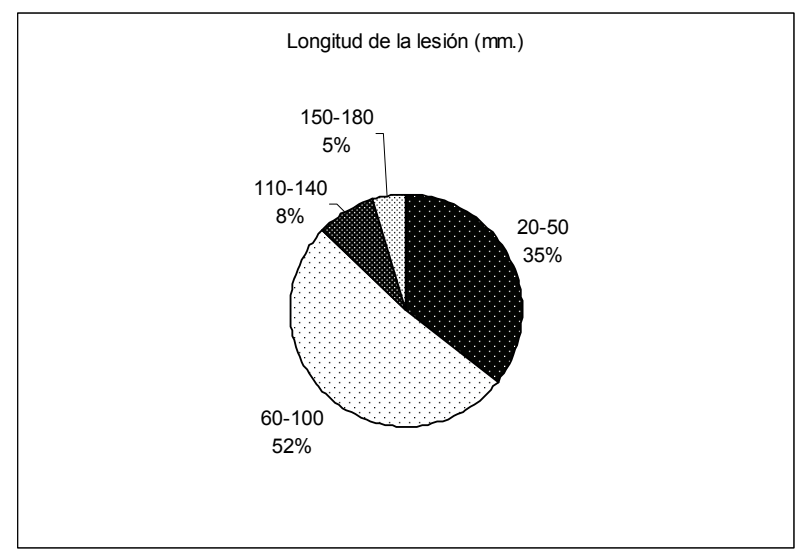

El resto de datos obtenidos tanto de la historia y exploración como de los resultados analíticos fueron completamente normales o en relación con otras patologías previas sin relación alguna con LS.

En la revisión a los 3 meses, casi un 75\% de los casos tienen evolución a la recuperación, mostrando remisión completa un 43,4\% y remisión parcial un 30,4 \%. No mostraron cambios un 26,2 \% (Figura 4).

Figura 4 - Evolución a los 3 meses

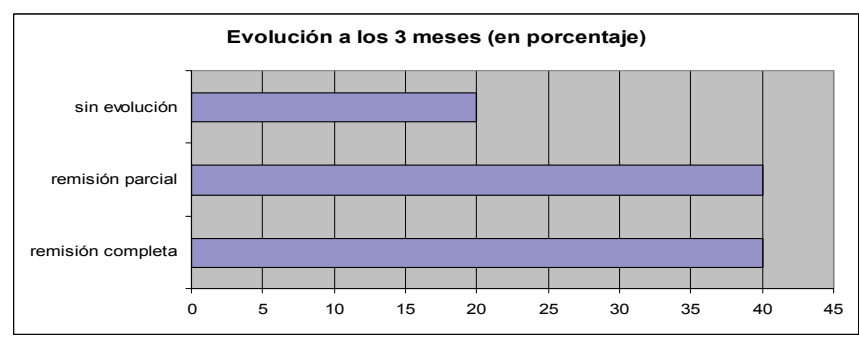

En las consultas de seguimiento encontramos que a los 6 meses un 93\% de pacientes había experimentado remisión (62\% completa, 31\% parcial) y 4 permanecían sin cambios. Al completar un año sólo 2 pacientes no habían presentado remisión alguna. (Figura 5). 
Figura 5 - Grado de remisión en lo sucesivos controles trimestrales

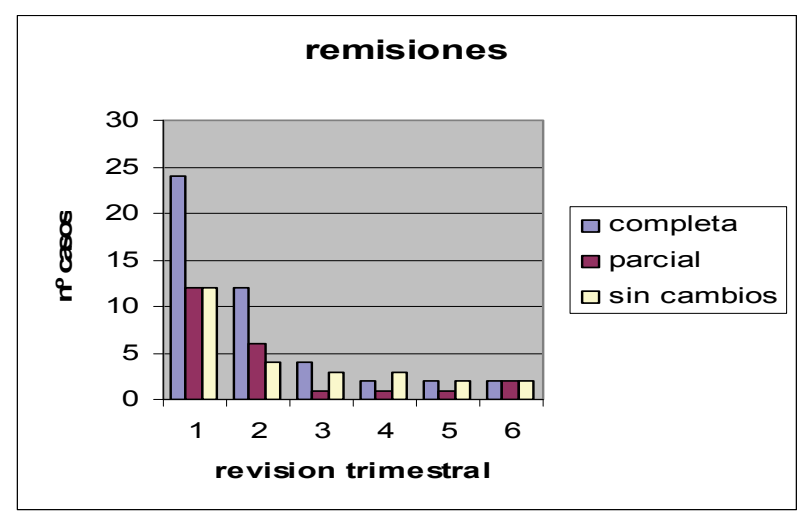

Finalmente el número de nuevos casos presentados ha ido disminuyendo de manera exponencial desde los 36 atendidos durante el primer año, a los 4 en lo que llevamos de este año (Figura 6)

Figura 6 - Número de casos nuevos anuales

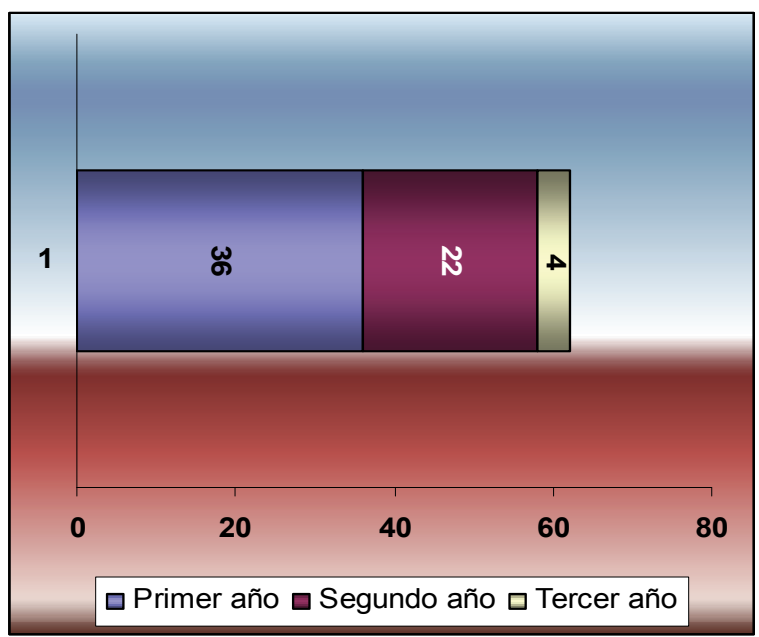

\section{DISCUSIÓN}

Determinadas condiciones medioambientales detectadas en algunos edificios "inteligentes", como unos valores elevados de electricidad estática junto a una baja humedad ambiental, tienen una gran relación con la aparición súbita de casos de LS.

En nuestra serie se confirman los resultados iniciales que publicamos tras el primer año ${ }^{24}$ en cuanto a que las características clínicas encontradas son similares en edad, sexo, localización y tipo de trabajador afecto (largas horas de escritorio con instrumental electrónico), a las series publicadas hasta ahora. Así mismo la intensidad de afectación muestra gran variabilidad, habiendo desde ligeras lesiones unilaterales a extensas bilaterales. La mayoría de los pacientes desconoce el momento de inicio, y por tanto el tiempo de evolución, al no tener sintomatología acompañante y ser un cuadro que se evidencia de forma accidental o provocado ante las recomendaciones efectuadas a partir de un primer caso.

Es evidente un gran predominio de la presentación en el sexo femenino, en relación probable con las distintas características anatómicas y estructurales del tejido graso entre hombres y mujeres ${ }^{25}$.

Las medidas de corrección aplicadas en los puestos de trabajo van reducen la carga electrostática (tomas de tierra, humedad relativa mínima del 50\%, recubrimiento de 
cableados, sillas antiestáticas, ropa de lana o algodón, zapatos de suela de goma, aumentar la distancia entre el trabajador y fuentes como monitores y CPUs, etc.) y han demostrado una gran eficacia ya que por un lado, la lesión va a revertir en un $97 \%$ de los casos diagnosticados en un período de entre 3 y 6 meses y por otro, se disminuye significativamente la aparición de nuevos casos. La resolución es más frecuente y más rápida que en los casos publicados anteriormente en los que no se modificaba el entorno laboral y solamente mejoraba cuando el paciente dejaba su puesto largos períodos de tiempo (bajas laborales por otras causas, jubilaciones).

Se trata de una enfermedad leve, reversible en casi todos los casos con unas medidas adecuadas, sin relación con factores como el tamaño o la lateralidad de la lesión y que cursa como única sintomatología, sin complicaciones sistémicas ni secuelas añadidas. En ningún caso se han detectado alteraciones en piel, músculo ni nervios locoregionales.

Hasta ahora no se ha mostrado ninguna otra afectación relacionada salvo el estrés inicial, que consideramos provocado por la alarma surgida tras la aparición de los primeros casos erróneamente etiquetados como una nueva patología provocada por el "síndrome del edificio enfermo". Una vez redimensionado su impacto como recomendábamos en nuestra publicación preliminar ${ }^{24}$, a base de tomar todas las medidas necesarias para evitar su extensión y de difundir una correcta y exhaustiva información al respecto entre la población, en particular entre la población laboral afectada y de riesgo, se ha mostrado una realidad de escaso significado patológico fácilmente corregible.

En el momento actual se debe insistir en las medidas preventivas conocidas, siempre considerando que muy probablemente estemos ante un cuadro cuya etiología y resolución sean multifactoriales, entre los que algunos condicionarían una especial sensibilidad en ciertos pacientes, necesitándose seguimiento a más largo plazo de los casos persistentes (e investigando el por qué de su mala evolución), estudios observacionales con un mayor número de casos y realización de estudios experimentales, como el llevado a cabo sobre las alteraciones en el DNA del adipocito producidas por los campos eléctricos, dosis dependientes, del Dr. Vershaeven ${ }^{25}$.

Finalmente resaltar que dentro de ser una enfermedad de poca entidad médica, adquirió gran eco social a su inicio hace 2 años, por sus importantes implicaciones económico/laborales al producirse en el centro de trabajo, en edificios vanguardistas con equipamientos muy costosos, trasmitiéndose rápidamente la sensación de una patología "epidémica". La realidad actual es que ha sido controlada en poco tiempo sin complicaciones y nuevamente limitada a su lugar correspondiente en el ámbito médico.

\section{AGRADECIMIENTOS}

Este trabajo ha sido posible gracias a la colaboración y esfuerzo inestimables de las Stas. Magdalena Herraiz Martínez y Francisca Vázquez Vázquez en el Hospital de día de Fraternidad-Muprespa en Madrid).

\section{REFERENCIAS}

1. Gschwandtner WR, Münzberger H. Lipoatrophia semicircularis. Ein Beitrag zu bandförmig-circulären Atrophien des subcutanen Fettgewebes im Extremitätenbereich. Der Hautartz 1974; 25: $222-227$.

2. Gschwandtner WR, Münzberger H. Lipoatrophia semicircularis. Wiener klein. Wochenschr. 1975; 87: 164-168).

3. Karkavitsas C, Miller JA, Kirby JD. Semicircular lipoatrophy. Br J Dermatol. 1981 Nov;105(5):591-3.

4. de Rie MA. Impressions on the thighs; semicircular lipoatrophy]. Ned Tijdschr Geneeskd. 1998 Apr 4;142(14):796-7.

5. Spencer DM, Parks AJ, Sydor LC, Pellegrini AE. Yellow nodule within an atrophic thigh patch. Localized lipoatrophy, semicircular type. Arch Dermatol. 1994 Aug;130(8):1056-7, 1059-60. 
6. Rongioletti F, Rebora A. Annular and semicircular lipoatrophies. Report of three cases and review of the literature. J Am Acad Dermatol. 1989 Mar;20(3):433-6.

7. Asherson RA, Shoenfeld Y. Annular, semicircular lipoatrophy of the thighs associated with incomplete "CREST" syndrome: Raynaud's, telangiectasias, and esophageal dysmotility. J Clin Rheumatol. 2003 Apr;9(2):129-33.

8. Haas N, Henz BM, Bunikowski R, Keitzer R. Semicircular lipoatrophy in a child with systemic lupus erythematosus after subcutaneous injections with methotrexate. Pediatr Dermatol. 2002 SepOct;19(5):432-5.

9.-Merelo, Alcocer, Rodríguez, Myrna Lipoatrofia localizada. Reporte de un caso, Revista central Dermatología Pascua, Vol. 12, núm. 1, enero-abril 2003, 31-33.

10. Herane MI, Urbina F, Sudy E. Lipoatrophia semicircularis: a compressive lipoatrophy consecutive to persistent mechanical pressure. J Dermatol. 2007 Jun;34(6):390-3.

11. Gómez-Espejo C, Pérez-Bernal A, Camacho-Martínez F. A new case of semicircular lipoatrophy associated with repeated external microtraumas and review of the literature. J Eur Acad Dermatol Venereol. 2005 Jul;19(4):459-61.

12. Kayikcioglu A, Akyurek M, Erk Y. Semicircular lipoatrophy after intragluteal injection of benzathine penicillin. J Pediatr. 1996 Jul;129(1):166-7.

13. Bloch PH, Runne U. [Lipoatrophia semicircularis in the male. Coincidence of arterial variations and microtraumas as a possible disease cause]. Hautarzt. 1978 May;29(5):270-2.

14. Imamura S, Taniguchi S. Lipoatrophic lesions preceded by pain and erythema a new clinical entity? Eur. J. Dermatol. 2000; 10: 540-541.

15. Maes A, Curvers B, Verschaeve L. Lipoatrophia semicircularis: the electromagnetic hypothesis. Electromagnetic Biology and Medicine 2003; 22 (2).

16. Cruceta G. Lipoatrofia semicircular. Efectos sobre la salud. Seminario Técnico Internacional. Madrid. 2008 Nov.

17. Zalla MJ, Winkelmann RK, Gluck OS. Involutional lipoatrophy: macrophage-related involution of fat lobules. Dermatology 1995; 191: 149-153.

18. Amaley I, Augsten K, Berg H. Electrostimulation of macrophage NADPH oxidase by modulated highfrequency electromagnetic fields. Bioelectrochem. Bioenerget. 1995; 38: 415-418).

19. Curvers Bart, Annemarie Maes, Luc Verschaeve Lipoatrophia semicircularis: An Electromagnetic Hypothesis. Electromagnetic Biology and Medicine, 2003, Volume 22, Issue 2\&3.

20. Flagothier C, Quatresooz P, Pierard GE. Electromagnetic lipolysis and semicircular lipoatrophy of the thighs]. Ann Dermatol Venereol. 2006 Jun-Jul;133(6-7):577-80.

21. Cruceta G. Síndrome de Lipoatrofia semicircular (LS) relacionado con los edificios. El Instalador, $\mathrm{N}^{\circ} 444$, 2007,114-121.

22. Senecal S, Victor V, Choudat D, Hornez-Davin S, Conso F. Semicircular lipoatrophy: 18 cases in the same company. Contact Dermatitis. 2000 Feb;42(2):101-2.

23. Ogino J, Saga K, Tamagawa M, Akutsu Y. Magnetic resonance imaging of semicircular lipoatrophy. Dermatology. 2004;209(4):340-1.

24. Cuevas F, Ortega A, Aguado E. Estudio clínico evolutivo de 30 nuevos casos de lipoatrofia semicircular. La Mutua. 2008; 19: 79-83.

25. Vershaeven L. Nuevas investigaciones y experiencia europea. Seminario Técnico Internacional. Madrid. 2008 Nov. 